\title{
A QUICK TEST FOR NONISOMORPHISM OF ONE-RELATOR GROUPS
}

\author{
K. J. HORADAM
}

\begin{abstract}
A sequence of integers is read off from the presentation of a finitely generated torsion-free one-relator group with nontrivial second integral homology, without recourse to group-theoretic manipulations. This test sequence is derived from the cup coproduct on the coring of the integral homology module of the group, and reflects information about the group's second lower central factor group.

Test sequences differ only if the corresponding groups are nonisomorphic. The test process can be generalised to any one-relator group with nontrivial second integral homology.
\end{abstract}

1. Introduction. It is generally difficult to determine when two one-relator groups are isomorphic (see [1, p. 75] for example), though S. J. Pride [7] has shown that the isomorphism problem for two-generator one-relator groups with torsion is solvable. This paper describes a test for nonisomorphism of one-relator groups with isomorphic integral homology modules. The test is directly applicable only to groups which are torsion-free, finitely generated and have nontrivial second integral homology, but may be extended to any one-relator group with nontrivial second integral homology.

The test's main advantages are that it is quick, that it involves arithmetic calculations only, rather than manipulation of group elements, and that the required test integers can be read off from any presentation of the group.

In $\$ 2$ the test is described and in $\$ 3$, proof that it measures nonisomorphism is given. In $\$ 4$ it is shown that the test may be extended to any one-relator group by comparison with a suitable group to which the test directly applies.

I would like to thank Drs. D. Collins, C. R. Leedham-Green and S. J. Pride for their helpful comments.

2. The test. This technique isolates a sequence of integers from a presentation of a torsion-free finitely generated one-relator group with nontrivial second integral homology (hereinafter referred to as a testable group). Sequences determined from two testable groups differ only if the groups themselves differ, in which case a specific homomorphism defined between their respective second lower central factor groups is not an isomorphism.

Received by the editors July 9, 1979.

1980 Mathematics Subject Classification. Primary 20E06, 20J05; Secondary 20 F 10.

Key words and phrases. One-relator group, isomorphism problem, lower central sequence, integral homology module, cup coproduct, Fox derivative, invariant factors of matrix. 
Two steps are involved: the first reads off a skew-symmetric integral matrix from a presentation of a testable group and the second derives from this matrix a test sequence of integers which is independent of isomorphisms of the group and hence is presentation-free.

In all that follows, let $G=\left\langle x_{1}, \ldots, x_{n}: r\right\rangle$ be a presentation of a testable group, let $F$ be the free group generated by $\left\{x_{1}, \ldots, x_{n}\right\}$, let

$$
1 \rightarrow R \rightarrow F \stackrel{\pi}{\rightarrow} G \rightarrow 1
$$

be the corresponding free presentation of $G$, and let $\varepsilon: \mathbf{Z F} \rightarrow \mathbf{Z}$ be the augmentation map of the group ring $\mathbf{Z} F$ over the integers $Z$. Recall that $\partial w / \partial z$ is the Fox derivative [2] in $\mathbf{Z F}$ of the element $w$ of $\mathbf{Z F}$ with respect to the generator $z$ of $F$.

(2.1) Definition. For each word $w$ and pair of generators $(y, z)$ in $F$, define the integer $\langle w ; y, z\rangle$ to be

$$
\langle w ; y, z\rangle=\varepsilon\left(\partial^{2} w / \partial y \partial z\right)
$$

That is, for $y \neq z,\langle w ; y, z\rangle$ is the exponent sum in $w$ of occurrences of $y$ preceding each occurrence of $z^{+1}$, minus the exponent sum in $w$ of occurrences of $y$ preceding each occurrence of $z^{-1}$. Thus $\left\langle y z^{-2} y^{2} z ; y, z\right\rangle=(-1)+(-1)+(3)=1$.

Recall further the definition of the invariant factors of an integral matrix. If $V$ is an $n \times n$ matrix with integer entries the ith determinantal divisor $d_{i}(V)$ of $V$ for $0<i<n$ is defined to be $d_{0}(V)=1$,

$$
d_{i}(V)=\operatorname{gcd}\{\operatorname{det} v: v \text { is an } i \times i \text { submatrix of } V\}, \quad 1<i<n .
$$

(2.2) Definition. The ith invariant factor $s_{i}=s_{i}(V)$ of $V$ is

$$
s_{i}(V)= \begin{cases}d_{i}(V) / d_{i-1}(V), & 1<i<\operatorname{rank} V, \\ 0, & \operatorname{rank} V<i<n .\end{cases}
$$

If $V$ is skew-symmetric with rank $V=2 l$ it is true (see [5, Theorems IV.2, IV.3] for example) that $s_{2 k}(V)=s_{2 k-1}(V) 1<k<l$.

These two definitions are all that is required to implement the test.

Test Step 1. (i) For each pair of generators $\left(x_{i}, x_{j}\right)$ with $i<j$ in $G$, calculate $\left\langle r ; x_{i}, x_{j}\right\rangle$.

(ii) Form the skew-symmetric matrix $M=M(G)$ with entries

$$
m_{k l}= \begin{cases}\left\langle r ; x_{k}, x_{l}\right\rangle, & k<l, \\ 0, & k=l, \\ -\left\langle r ; x_{l}, x_{k}\right\rangle, & k>l .\end{cases}
$$

Test Step 2. Calculate the invariant factors $s_{i}(M(G)), 1<i<n$. The sequence of invariant factors $T S(G)=\left(s_{1}, s_{2}, \ldots, s_{n}\right)$ is the required test sequence for $G$. If the test sequences for two presentations differ at any co-ordinate then they are presentations of nonisomorphic groups.

Computation in Test Step 1(i) may be simplified if a representation $r \equiv$ $r^{*} \bmod F_{3}$ is known, where

$$
r^{*}=\prod_{j=2}^{n} \prod_{i<j}\left[x_{i}, x_{j}\right]^{m_{i j}}
$$


If $\left\langle r^{*},\left[x_{i}, x_{j}\right]\right\rangle$ denotes the exponent sum in $r^{*}$ of commutator $\left[x_{i}, x_{j}\right]$ then the identity

$$
\left\langle r ; x_{i}, x_{j}\right\rangle=\left\langle r^{*},\left[x_{i}, x_{j}\right]\right\rangle, \quad 1<i<j<n,
$$

may be used; that is, $\left\langle r ; x_{i}, x_{j}\right\rangle=m_{i j}, 1<i<j<n$. Note this implies that any skew-symmetric $n \times n$ integral matrix $V$ appears as $V=M(G)$ for at least one testable group $G$.

In illustration, consider $G=\left\langle x_{1}, x_{2}, x_{3}, x_{4}: r\right\rangle$ where

$$
r=\left[x_{3}, x_{4}\right]\left[x_{1}, x_{2}\right]\left[x_{1}, x_{4}^{2}\right]\left[x_{3}, x_{2}\right]\left[x_{2}, x_{4}\right]\left[x_{3}, x_{4}\right]^{3}
$$

and $H=\left\langle x_{1}, x_{2}, x_{3}, x_{4}: s\right\rangle$ where

$$
s=r\left[x_{4}, x_{3}\right]
$$

Then

$$
M(G)=\left[\begin{array}{rrrr}
0 & 1 & 0 & 2 \\
-1 & 0 & -1 & 1 \\
0 & 1 & 0 & 4 \\
-2 & -1 & -4 & 0
\end{array}\right] \text { and } M(H)=\left[\begin{array}{rrrr}
0 & 1 & 0 & 2 \\
-1 & 0 & -1 & 1 \\
0 & 1 & 0 & 3 \\
-2 & -1 & -3 & 0
\end{array}\right]
$$

Since $d_{1}(M(G))=1$ it follows that $s_{1}(M(G))=s_{2}(M(G))=1$, and as $d_{4}(M(G))=$ $\left[d_{3}(M(G))\right]^{2}=4$, it follows that $s_{3}(M(G))=s_{4}(M(G))=2$ and $T S(G)=$ $(1,1,2,2)$. Similarly, $T S(H)=(1,1,1,1)$, hence $G \approx H$.

(2.3) ExAmple. Let $G=\left\langle x_{1}, x_{2}: r\right\rangle$ be a testable two-generator group. Then $r \equiv\left[x_{1}, x_{2}\right]^{p} \bmod F_{3}$ for a unique integer $p$, so that $T S(G)=(|p|,|p|)$, thus there are infinitely many distinct isomorphism classes of such groups.

(2.4) EXAMPLE. Let $G=\left\langle x_{1}, x_{2}, x_{3}: r\right\rangle$ be a testable three-generator group. Then $r \equiv\left[x_{1}, x_{2}\right]^{k}\left[x_{1}, x_{3}\right]^{l}\left[x_{2}, x_{3}\right]^{m} \bmod F_{3}$ and $T S(G)=(g, g, 0)$ where $g=(k, l, m)$. Thus there are infinitely many distinct isomorphism classes of such groups.

(Analysis similar to that of the prior two examples clearly holds for groups with any number of generators; for instance there is a doubly-countable number of distinct isomorphism classes of testable five-generator groups.)

3. The homomorphism $\mathscr{D}(G)$. We prove that the test sequence $T S(G)$ measures (in a way to be made precise) the second term $G_{2} / G_{3}$ of the lower central factors of $G$. Recall that for a testable group $G, H_{2}(G ; Z) \cong Z$ and $H_{1}(G ; Z)$ is free abelian.

Let $\eta: R \cap F_{2} /[R, F] \rightarrow H_{2}(G ; Z)$ be the Hopf isomorphism, $\mu: F_{2} / F_{3}[R, F] \rightarrow$ $\wedge_{2} H_{1}(G ; Z)$ be the isomorphism given by $\left[f, f^{*}\right] F_{3}[R, F] \rightarrow \pi(f) G_{2} \wedge \pi\left(f^{*}\right) G_{2}$, [ , ]: $\wedge_{2} H_{1}(G ; Z) \rightarrow G_{2} / G_{3}$ be the homomorphism given by $g G_{2} \wedge g^{*} G_{2} \rightarrow$ $\left[g, g^{*}\right] G_{3}$, and $\iota: R \cap F_{2} /[R, F] \rightarrow F_{2} / F_{3}[R, F]$ be the homomorphism induced by inclusion.

(3.1) Definition. The homomorphism $\mathscr{D}(G): H_{2}(G ; Z) \rightarrow \bigwedge_{2} H_{1}(G ; Z)$ is defined to be $\mathscr{D}(G)=\mu \circ \imath \circ \eta^{-1}$.

In [3] it is shown firstly that the sequence

$$
H_{2}(G ; Z) \stackrel{\mathscr{D}(G)}{\rightarrow} \bigwedge_{2} H_{1}(G ; Z) \stackrel{[,]}{\rightarrow} G_{2} / G_{3} \rightarrow 1
$$


is exact, secondly that

$$
\mathscr{D}(G) \circ \eta(r[R, F])=\sum_{j=2}^{n} \sum_{i=1}^{j-1}\left\langle r ; x_{i}, x_{j}\right\rangle\left(x_{i} G_{2} \wedge x_{j} G_{2}\right),
$$

and thirdly that $\mathscr{D}(G)=\phi \circ \Omega(G)$ where $\Omega(G)$ is the diagonal comultiplication (cup coproduct)

$$
\Omega(G): H_{2}(G ; \mathbf{Z}) \rightarrow H_{1}(G ; \mathbf{Z}) \nabla H_{1}(G ; \mathbf{Z}) \subseteq H_{1}(G ; \mathbf{Z}) \otimes H_{1}(G ; \mathbf{Z})
$$

and $\phi$ is the isomorphism defined by

$$
\left(x_{i} G_{2} \otimes x_{j} G_{2}-x_{j} G_{2} \otimes x_{i} G_{2}\right) \rightarrow x_{i} G_{2} \wedge x_{j} G_{2}, \quad 1<i<j<n .
$$

Suppose $G$ and $H$ are testable groups with isomorphic integral homology modules. If $\alpha: H_{2}(G ; Z) \rightarrow H_{2}(H ; Z)$ is an isomorphism (so $\alpha= \pm 1$ ) and $\beta$ : $H_{1}(G ; Z) \rightarrow H_{1}(H ; Z)$ is any isomorphism, it is easy to deduce that

$$
(\beta \otimes \beta) \circ \Omega(G)=\Omega(H) \circ \alpha \Leftrightarrow(\beta \wedge \beta) \circ \mathscr{D}(G)=\mathscr{D}(H) \circ \alpha .
$$

When isomorphisms $\alpha$ and $\beta$ exist satisfying these conditions, we write $\Omega(G) \sim$ $\Omega(H)$ via $(\alpha, \beta)$, or, equivalently, $\mathscr{D}(G) \sim \mathscr{D}(H)$ via $(\alpha, \beta)$, and $\sim$ is an equivalence relation on the set of such homomorphisms.

Since $\Omega(G) \sim \Omega(H)$ via $(\alpha, \beta)$ if and only if $M(H)= \pm B^{\top} M(G) B$, where $B$ is the change-of-basis matrix corresponding to $\beta$, we conclude that $\Omega(G) \sim \Omega(H)$ if and only if $M(G)$ and $M(H)$ have the same invariant factors (for instance, see [5, Theorem IV.3]). This specifies the connection between the test sequence $T S(G)$ and the homomorphism $\mathscr{D}(G)$ :

$$
T S(G)=T S(H) \Leftrightarrow \mathscr{D}(G) \sim \mathscr{D}(H) .
$$

A preliminary definition is needed before the relationship between $\mathscr{D}(G)$ and $G_{2} / G_{3}$ may be described. Any isomorphism $\beta: H_{1}(G ; Z) \rightarrow H_{1}(H ; Z)$ induces a set map $\beta^{\prime}: G_{2} / G_{3} \rightarrow H_{2} / H_{3}$ given by

$$
\beta^{\prime}\left(\prod_{i=1}^{n}\left[g_{i}, g_{i}^{\prime}\right]^{s_{i}} G_{3}\right)=\prod_{i=1}^{n}\left[h\left(g_{i}\right), h\left(g_{i}^{\prime}\right)\right]^{s_{i}} H_{3}
$$

where $h(g)$ is a coset representative of $\beta\left(g G_{2}\right)$. It follows that

$$
[,] \circ(\beta \wedge \beta)=\beta^{\prime} \circ[,] \text {. }
$$

(3.2) TheOrem. Let $G$ and $H$ be testable groups and let $\beta$ : $H_{1}(G ; Z) \rightarrow H_{1}(H ; Z)$ be an isomorphism. Then $\mathscr{D}(G) \sim \mathscr{D}(H)$ via $(\alpha, \beta)$ if and only if $\beta^{\prime}: G_{2} / G_{3} \rightarrow$ $\mathrm{H}_{2} / \mathrm{H}_{3}$ is an isomorphism.

Proof. Consider the commuting diagram

$$
\begin{array}{ccccccc}
H_{2}(G ; \mathbf{Z}) & \stackrel{\mathscr{D}(G)}{\rightarrow} & \bigwedge_{2} H_{1}(G ; \mathbf{Z}) & \stackrel{[,]}{\rightarrow} & G_{2} / G_{3} & \rightarrow & 1 \\
& & \beta \wedge \beta \downarrow & \circ & \downarrow \beta^{\prime} & & \\
H_{2}(H ; Z) & \underset{\mathscr{D}(H)}{\rightarrow} & \bigwedge_{2} H_{1}(H ; Z) & \rightarrow,] & H_{2} / H_{3} & \rightarrow & 1
\end{array}
$$

If $\mathscr{D}(G) \sim \mathscr{D}(H)$ via $(\alpha, \beta)$ then $\beta^{\prime}$ is an isomorphism immediately. Conversely, if $\beta^{\prime}$ is an isomorphism, then since (in either row) $\operatorname{Ker}[$,$] is unique up to$ 
isomorphism, there exists an isomorphism $\alpha: H_{2}(G ; Z) \rightarrow H_{2}(H ; Z)$ such that $(\beta \wedge \beta) \circ \mathscr{D}(G)=\mathscr{D}(H) \circ \alpha$.

(3.3) CoROllary. If $G$ and $H$ are testable groups with isomorphic integral homology then $\operatorname{TS}(G)=T S(H)$ if and only if there exists an isomorphism $\beta$ : $H_{1}(G ; Z) \rightarrow H_{1}(H ; Z)$ inducing an isomorphism $\beta^{\prime}: G_{2} / G_{3} \rightarrow H_{2} / H_{3}$.

Finally, suppose $\gamma: G \rightarrow H$ is an isomorphism of testable groups. Then $\gamma$ induces isomorphisms $\gamma_{a b}: H_{1}(G ; Z) \rightarrow H_{1}(H ; Z)$ and $\gamma_{2}: G_{2} / G_{3} \rightarrow H_{2} / H_{3}$ with $\gamma_{2}=\gamma_{a b}^{\prime}$ necessarily. Hence

$$
T S(G) \neq T S(H) \Rightarrow G \approx H .
$$

\section{General use of the test.}

(4.1) Groups with torsion. (See [6, Corollary 4.13.1], for example.) If $G$ and $H$ are torsion-free one-relator groups with $G=\left\langle x_{1}, \ldots, x_{n}: r\right\rangle$ and $H=$ $\left\langle x_{1}, \ldots, x_{n}: s\right\rangle$ and if $G(k)=\left\langle x_{1}, \ldots, x_{n}: r^{k}\right\rangle$ and $H(k)=\left\langle x_{1}, \ldots, x_{n}: s^{k}\right\rangle$ then

$$
G \approx H \Rightarrow G(k) \approx H(k) \text { for all } k \in \mathbf{Z} .
$$

(4.2) Infinitely generated groups. If $G^{*}$ is an infinitely generated one-relator group it can be decomposed as a free product $G^{*}=G * G^{\prime}$ where $G$ is a finitely-generated one-relator group on a minimum number of generators and $G^{\prime}$ is free on the set $X(G)$. If $H^{*}=H * H^{\prime}$ is another such decomposition, the Kurosh decomposition theorem $[4, \S 35]$ implies

$$
G^{*} \cong H^{*} \Leftrightarrow G \cong H \text { and } \operatorname{card} X(G)=\operatorname{card} X(H),
$$

so in particular $G \cong H \Rightarrow G^{*} \nRightarrow H^{*}$.

This analysis also applies to finitely generated groups not presented on a minimal generating set.

(4.3) Groups with trivial second integral homology. If $G^{*}$ is a one-relator group with $H_{2}\left(G^{*} ; Z\right)=0$ it may be embedded in a one-relator group $G$ with $\mathrm{H}_{2}(G ; Z)$ $\neq 0$ in such a fashion that if $H^{*}$ is another such group then in certain cases $G \approx H$ implies $G^{*} \approx H^{*}$.

Let $G^{*}=\left\langle x_{1}, \ldots, x_{n}: r^{*}\right\rangle$ where $r^{*}$ is cyclically reduced of length $>1$ and $n>2$. If $F^{*}$ is the free group on $\left\{x_{1}, \ldots, x_{n}\right\}$ then $r^{*} \notin F_{2}^{*}$ and thus some generator of $F^{*}$ has nonzero exponent sum in $r^{*}$. By [6, Theorem 3.5] it may be assumed that only one generator ( $x_{1}$ say) has nonzero exponent sum ( $\alpha$ say) in $r^{*}$. Then $G^{*}$ can be embedded in a one-relator group $G=\left\langle a, b, x_{2}, \ldots, x_{n}: r\right\rangle$ in which every generator has zero exponent sum in $r$. In fact $G \cong G^{*}{ }_{A} Y$ where $Y$ is the free group on $\{a, b\}$ and the (free) subgroup $A$ of $G^{*}$ generated by $\left\{x_{1}\right\}$ is amalgamated by the isomorphism $x_{1} \rightarrow[a, b]$. If $G^{*}$ is torsion-free then so is $G$ and $H_{2}(G ; Z) \cong Z$. Finally, suppose $H^{*}$ is another such group with isomorphic integral homology. Necessarily $H^{*}$ has a presentation $\left\langle x_{1}, \ldots, x_{n}: s^{*}\right\rangle$ where $x_{1}$ is the only generator with exponent sum nonzero (in fact equal to $\alpha$ ). If $G \approx H$, then one of two conclusions may be drawn: either $G^{*} \approx H^{*}$, or else $G^{*} \approx H^{*}$ but no isomorphism $\phi: G^{*} \rightarrow H^{*}$ has $\phi\left(x_{1}\right)=x_{1}^{ \pm 1}$. 
5. Concluding remarks. Since $\operatorname{Ker} \iota=R \cap F_{3}[R, F] /[R, F]$ for a testable group $G$, it follows that $\mathscr{D}(G) \sim 0$ if and only if $r \in F_{3}$. Equivalently, if $H=$ $\left\langle x_{1}, \ldots, x_{n}: s\right\rangle$ is another testable group, then $\mathscr{D}(G)=\mathscr{D}(H)$ if and only if $r \equiv s \bmod F_{3}$, or, in other words, $T S(G) \neq T S(H)$ only if $r \neq s \bmod F_{3}$. Thus the test sequence is not sufficient to distinguish between one-relator groups. For instance, if $G=\left\langle x_{1}, x_{2}:\left[x_{1}, x_{2}^{4}\right]\right\rangle$ and $H=\left\langle x_{1}, x_{2}:\left[x_{1}^{2}, x_{2}^{2}\right]\right\rangle$, then $T S(G)=$ $T S(H)$, but $G \supsetneqq H$ since $G$ has infinite cyclic centre and $H$ has trivial centre.

However, the test sequence for $G$ does generally carry more information than is given by $G_{2} / G_{3}$, by virtue of Corollary (3.3). In fact, if the relator $r$ of the testable group $G$ is written in the form $r \equiv r^{*} \bmod F_{3}$ specified earlier, then the relation matrix of the abelian group $G_{2} / G_{3}$ is the single column matrix $\left(\left\langle r^{*},\left[x_{i}, x_{j}\right]\right\rangle\right)$ with $1 \leqslant i<j \leqslant n$. Hence $G_{2} / G_{3} \cong \mathbf{Z} /\langle g\rangle \oplus \mathbf{Z}^{m-1}$, where $m=\frac{1}{2} n(n-1)$ and $g=$ $\operatorname{gcd}\left\{\left\langle r^{*},\left[x_{i}, x_{j}\right]\right\rangle, 1 \leqslant i<j \leqslant n\right\}$. Because $T S(G)=\left(g, g, s_{3}, \ldots, s_{n}\right)$ it follows that for another testable group $H, G_{2} / G_{3} \simeq H_{2} / H_{3}$ if and only if $T S(G)$ and $T S(H)$ agree on their first two coordinates.

\section{REFERENCES}

1. G. Baumslag, Some problems on one-relator groups (Proc. Second Internat. Conf. Theory of Groups, Canberra, 1973), Lecture Notes in Math., Vol. 372, Springer-Verlag, Berlin, 1974, pp. 75-81.

2. R. H. Fox, Free differential calculus. I, Ann. of Math. (2) 57 (1953), 547-560.

3. K. J. Horadam, The diagonal comultiplication on homology, J. Pure Appl. Algebra (to appear).

4. A. G. Kurosh, The theory of groups, Vol. II, Chelsea, New York, 1956.

5. M. Newman, Integral matrices, Academic Press, New York, 1972.

6. W. Magnus, A. Karrass and D. Solitar, Combinatorial group theory, 2nd rev. ed., Dover, New York, 1976.

7. S. J. Pride, The isomorphism problem for two-generator one-relator groups with torsion is solvable, Trans. Amer. Math. Soc. 227 (1977), 109-139.

School of Mathematical and Physical Sciences, Murdoch University, Murdoch, W.A. 6150, Australia

Current address: Department of Mathematics, Monash University, Clayton, Victoria 3168, Australia 